\title{
EL EMPRENDIMIENTO SOCIAL: UN VALOR ORGANIZACIONAL
}

\author{
Amalfi Babilonia Zambrano' \\ Carmen Luz de Pretelt ${ }^{2}$
}

Recibido: 16 de mayo de 2013 Aceptado: 12 de agosto de 2013

\section{Resumen}

En el presente artículo se reflexiona sobre la importancia del emprendimiento como un hecho esencial en el desarrollo humano y por lo tanto como un valor fundante e igualmente esencial en la organización, gestión y desarrollo académico para la formación integral en la UNAD. De manera somera se habla sobre el desarrollo del emprendimiento como parte del sector productivo en Colombia y se muestra como desde la epistemología del modelo pedagógico de la universidad, de los postulados de la educación a distancia, la educación permanente y la formación integral, el emprendimiento es raíz esencial para el desarrollo de dichos postulados. A la vez se mencionan casos experienciales de promoción y fortalecimiento del emprendimiento para la productividad y del desarrollo social vividos en la universidad, en la Escuela de Ciencias de la Administración, Contables y de Negocios - ECACEN y en el CEAD Simón Bolívar en Cartagena de Indias.

Palabras Claves: emprendimiento, desarrollo humano, formación integral, productividad, creatividad, innovación, gestión organizacional.

\section{SOCIAL ENTREPRENEURSHIP: AN ORGANIZATIONAL VALUE}

\section{Abstract}

In this article we examine the importance of entrepreneurship as an essential element in human development and further more as an essential foundational value in the institutions and as such, in the organization, management and the educational process in UNAD. In the article it is shown some aspects of how in Colombia has been developed the promotion for the entrepreneurship attitude. At the same time it is analyzed the essential root that entrepreneurship

1 Amalfi Babilonia Zambrano Administradora de Empresa-U. Simón Bolívar, Especialista en PedagogíaUNAD, Magister en Administración de Negocios-UNAD Florida, E-mail: amalfi.babilonia@unad.edu.co. 
value means for the development of the university postulates. And how all starts since UNAD's pedagogical model epistemology, reinforced by the postulates of distance education, continuing education and integral formation. At the same time it is shown how in the given praxis this value have been worked and lived at the university, and how it has been promoted from UNAD's Administration, Counting and Economical Sciences School and at the Simón Bolívar, Cartagena de India's Center for Distance Education.

Key Words: entrepreneurship, human development, holistic education, comprehensive training, productivity, creativity, organizations activities and innovation.

\section{Introduccion}

La sociedad de hoy requiere personas formadas para ejercer una ciudadanía responsable, que les permita desarrollarse integralmente como seres humanos: que les de plena libertad para crear y participar de manera activa y significativa en las transformaciones de su entorno y que les brinde las herramientas para desempeñarse con eficiencia en un mundo caracterizado por nuevas y mas complejas exigencias en materia laboral y productiva (Campo, 2011: 3).

Cuando se habla de emprendimiento se está tocando un tema de vital trascendencia para el desarrollo humano. La Psicología en sus estudios sobre el desarrollo humano desde lo biológico, desde lo funcional, desde el desarrollo evolutivo así como cognitivo, muestra como en el ser humano la competencia para emprender acciones es base de su propia supervivencia y desarrollo personal. Si un infante no tuviera esa capacidad de emprender acciones y movimientos, moriría. Su desarrollo físico, emocional, cognitivo depende en un $100 \%$ de esa competencia que lo lleva hacia la acción. Emprender por lo tanto, es eje fundamental para la supervivencia personal, ser productivo es esencia de la propia vida personal, y se refleja en los modos y formas en que el ser humano y las comunidades se organizan para la vida. Por lo tanto el emprendimiento se ve reflejado en la iniciación y puesta en marcha, de cada nueva idea, de cada nuevo proyecto, de cada gestión empresarial o de desarrollo social que surge como resultado de la actividad humana.

El ser humano se mueve hacia la acción, motivado por diferentes conceptos a los que les da un valor determinado; acciones que considera constituirán una cadena de valor agregado a su propia vida. Siendo las organizaciones resultado de esos valores motivantes de las personas, cada organización igualmente se constituye y funciona a partir de un cúmulo de valores (Bennink, 2010); valores que en algún momento dado, mueven a la o las personas que deciden constituirla, hacia la decisión -acción. Y aunque no hay organización que nazca sin la motivación para emprender, no implica esto que necesariamente el emprendimiento en si mismo, haya sido o siga siendo un valor central de la gestión de todas las organizaciones. Es decir el valor del emprendimiento se ve reflejado siempre en la constitución de una organización, mas quienes las crean puede ser que no consideren el emprendimiento como uno de sus ejes centrales de actuación.

Son esos valores centrales, la razón de ser de la institución, el motor para la gestión de la organización, el corazón del funcionamiento adecuado de cualquier entidad. Razón por la cual, es de suma importancia conocerlos, compren- 
derlos, valorarlos, institucionalizarlos y ponerlos continuamente en acción, con el fin de que cualquier organización cumpla con la razón de ser de su existencia y logre ser exitosa en los propósitos fundantes (Huettman, 2012).

En ese sentido, el de profundizar sobre los valores fundantes de la UNAD, y considerando que la formación para el emprendimiento es esencial en la formación integral que esta universidad pretende ofrecer a su comunidad académica, en este artículo se pretende realizar una reflexión sobre como se refleja el valor del emprendimiento en ella, para lo cual se analiza someramente la situación en Colombia, como país que sirve de contexto inicial de la gestión de la universidad, se reflexiona sobre las razones determinantes para considerar el valor del emprendimiento social como uno de los valores fundamentales de la razón de ser de la universidad, ya que el valor de emprender acciones con el fin de generar una mejora social, fue y sigue siendo eje vital de la gestión de la institución y por último se presentan estrategias organizacionales concretas, que muestran como emprender e innovar motivan la gestión de la UNAD para lograr cada vez más, una continúa inclusión social para todos los ciudadanos colombianos y globales, ofreciendo servicios educativos integrales, de máxima calidad.

\section{El emprendimiento social en Colombia}

Para entender la dinámica del emprendimiento social en Colombia, es necesario remontarse casi a los albores del siglo XVII; en efecto, los diversos grupos sociales de la época - indígenas, esclavos, comuneros, campesinos, artesanos y en general aquellos colectivos apabullados por la corona, los españoles y los criollos, conformaron una serie de movimientos sociales. Paralelo a las corrientes libertarias y de equidad; también se dieron organizaciones de corte más pacifista derivadas de las organizaciones indígenas tradicionales como la minga y el convite que, a través del trabajo comunitario, ejecutaban labores de construcción de casas, templos y caminos o bien en faenas agrícolas y de carácter económico (RedSUMA, 2012: 2).

En Colombia se estima que en el año 2010, existe un total de 10.866 entre cooperativas, fondos de empleados y sociedades mutuales que integran a más de 6 millones de afiliados según datos de Dansocial, entidad del Estado que regula el sector cooperativo en Colombia, mientras que se forman otras organizaciones sociales, las fundaciones, algunas investigaciones hablan de aproximadamente 120 fundaciones empresariales en, algunas de ellas se fundamenta el logro de realizar un trabajo social.

El Plan Nacional de Desarrollo 2010-2014: «Igualdad de oportunidades para la Prosperidad social», contempla: que el crecimiento económico conduce a un desarrollo social y la igualdad de oportunidades, se traducen en un bienestar social y mejora las condiciones de vida de la población (PND, 2010-2014).

El emprendimiento en Colombia, según la Ley 1014 de 2006, «es una forma de pensar, razonar y actuar centrada en las oportunidades, planteada con visión global y llevada a cabo mediante un liderazgo equilibrado y la gestión de un riesgo calculado, su resultado es la creación de valor que beneficia a la empresa, la economía y la sociedad; la misma Ley fomenta a la cultura del emprendimiento en Colombia, define ésta como el "conjunto de valores, creencias, ideologías, hábitos, costumbres y normas que comparten los individuos de una organización, los cuales generan patrones de comportamiento colectivos"» (Ley 1014 de 2006, art. 1º).

Es importante anotar que el emprendimiento social en Colombia, tiene sus avances, debido que desde distintas áreas se vienen realizando por 
profesionales, como proyecto de vida, la cual permite ayudar a los demás.

Uno de los emprendedores más reconocidos a nivel internacional, es Juan David Aristizábal, presidente de la Fundación Idea por un País mejor y Director de la fundación Buena Nota, se ha destacado por sus acciones en emprendimiento social. De igual forma existen en el país muchas entidades gubernamentales y no gubernamentales que impulsan el emprendimiento social.

\section{El emprendimiento en la UNAD}

Frente a los cambios del mundo contemporáneo, es inminente repensar la Universidad, para transformarla en respuesta apropiada al reto que tiene con el futuro, en un mundo globalizado, caracterizado por la sociedad del conocimiento, el avance de las tecnologías de la información y la comunicación, el crecimiento de las economías de mercado, los nuevos convenios comerciales, la apertura internacional y diversos escenarios que demandan nuevos conocimientos, mediante una educación más significativa para la vida del Siglo XXI (Leal, 2006: 213).

En la UNAD es fundamental que toda su comunidad académica tenga claro la importancia de desarrollar sus propias competencias para emprender, y así ser capaces de generar acciones que le permitan a cada uno de los integrantes de sus estamentos, desarrollar su autonomía y propiciar la propia capacidad de aprendizaje y de puesta en práctica de acciones pertinentes para el desarrollo humano, comunitario y social, a partir de una productividad que permee de forma pertinente la gestión que en la universidad se desarrolla. Para lograrlo en la universidad se vive esta realidad desde diferentes enfoques.

\section{La perspectiva epistemológica}

El Proyecto Pedagógico Solidario de la UNAD, se ha ido actualizando a través de los tiempos, sin embargo desde el inicio de la gestión educativa, ha tenido como fundamento la teoría constructivista, en la que se considera que el sujeto construye su conocimiento desde sus propios paradigmas, como resultado de un aprendizaje compartido culturalmente y apoyado en convenciones sociales. (Piaget, 1947, Vygotsky, 1934 \& Brunner, 1984). Es decir no recibe pasivamente la información sino que a partir de la investigación y de la profundización, y realizando un aprendizaje autónomo, construye su saber (UNISUR, 1986).

Esta construcción de saber no la realiza de manera aislada sino que además debe emprender acciones hacia la interacción social, para ir construyendo la realidad de manera compartida con otros, en continuos intercambios del intelecto y de la praxis. (Vygotsky, 1934 \& Brunner, 1984). En la UNAD no se pretende formar a la comunidad desde un postulado pasivo, sino se busca a partir de ofrecer servicios educativos integrales, que la comunidad educativa emprenda de manera decidida, un quehacer por el logro de su propia formación. De ahí que se pretenda el que los estudiantes y los académicos de la UNAD, no sean personas pasivas o sólo receptivas, sino que se espera y se aspira a que se mantengan en continuo movimiento, emprendiendo acciones para el fortalecimiento de su desarrollo personal, profesional y comunitario (UNAD, 2011).

\section{El emprendimiento en la misión y visión}

El valor del emprendimiento social se ve también reflejado tanto en la Misión como en la Visión de la UNAD, que nos muestran como se busca contribuir a la educación para todos. ¿Pero cómo? A partir de la investigación, de la acción pedagógica y de las innovaciones metodológicas, con el fin de acompañar a los estudiantes en su aprendizaje autónomo y en su capacidad de transformar a las comunidades y no de cualquier manera sino con calidad, eficiencia y equidad social. 
Pretende la UNAD ser una organización líder en Educación Abierta y a Distancia por su trabajo con calidad innovadora y por el aporte que se realice a las comunidades locales y globales. La invitación que llega a quienes se acercan a la UNAD, es a la acción investigativa e innovadora, para el cumplimiento de nuestras labores, ya sea como funcionarios o como estudiantes (Estatuto Organizacional, Acuerdo 0030 de marzo de 2012).

\section{El emprendimiento social y el componente organizacional}

La UNAD considera que como organización es el resultado del conjunto de relaciones entre las personas que la conforman. La organización de la UNAD se caracteriza por su complejidad sistémica que exige dinámicas innovadoras para poner en práctica la capacidad de aprendizaje, la capacidad de autorregulación y la capacidad de innovar para cumplir los propósitos misionales de acuerdo a las exigencias contextuales en que la UNAD desarrolla su labor educativa y social comunitaria (Leal, 2004).

La complejidad organizacional en la UNAD, exige de funcionarios que sean capaces de auto gestionar su propio aprendizaje y de promover formas superiores de aprender a autoconstruirse y a autorregularse, para que la organización se convierta en un ente inteligente en si mismo, que permita la continua innovación; para que adaptándose a los tiempos, la UNAD sea capaz de seguir ofreciendo servicios educativos de calidad, que apunten a la inclusión social, a la equidad y a cumplir con la misión de educación para todos, conjugando la equidad social con la modernización (UNAD, 2012).

La UNAD se encuentra ubicada en todas las regiones del país y reconoce la gran diversidad de culturas, de etnias y de formación cultural, según las diferentes regiones de Colombia. Situación que obliga a su comunidad académica en general, a desarrollar cambios mentales que permitan la inclusión en sus actividades académico-administrativas. Cambios mentales y cambios organizacionales, que llevan a su comunidad educativa a emprender acciones innovadoras para poder atender la variedad compleja de la ciudadanía colombiana y de la realidad global que se vive en esta sociedad del conocimiento del Siglo XXI.

\section{El emprendimiento social, la educación a dis- tancia y la educación permanente}

Los postulados de la educación a distancia exigen el desarrollo de una cultura emprendedora que permita a la comunidad académica desarrollar una estructura mental ampliada y con visión holista, integral y compleja. Una comunidad educativa dispuesta a entender y a aprender que no es en lo estático y paradigmático que se avanza sino a partir de la capacidad de emprendimiento y de innovación que nos ayudan a ubicarnos en la realidad contextualizada, «para impulsar, transformar y enriquecer, mediante la acción académica, la creatividad social, la investigación científica y la sistematización de experiencias como estrategias válidas de conocimiento» (Leal, 2004).

Otra constante que lleva a entender como el emprendimiento social es una variable, un valor inserto en la misma esencia de la UNAD, es el principio de la educación permanente, el cual se fundamenta en la capacidad inherente al ser humano del aprendizaje continuo y por siempre; por lo tanto, a la capacidad que tiene el ser humano para emprender y aceptar el cambio y la ampliación continua y constante del conocimiento (Ramón, 2004 \& Leal, 2012).

Ese principio de la educación permanente entraña una forma de vida que exige la investigación y la innovación continua para poderse adaptar como organización y para poder ofrecer un servicio que se encuentre inserto en las realidades no solo regionales sino también temporales. Es decir, de acuerdo con la época en que 
se desarrolla la gestión de la universidad y de acuerdo con la vida moderna que se actualiza y se transforma culturalmente y a partir de los medios y las mediaciones que se utilizan para ofrecer educación de calidad. De forma tal que su comunidad académica pueda insertarse de manera adecuada a la sociedad a la cual pertenece (Leal, 2012).

\section{El emprendimiento, eje de la formación integral} El componente académico y pedagógico de la universidad, es el fundamento, la razón de ser de la institución y el hilo conductor del que se desprende todo su accionar. Como se muestra en documentos del 2004 de la UNAD, y se reafirman en los documentos del Proyecto Pedagógico Solidario en el 2011, la universidad se mueve para cumplir tres voluntades en forma integrada. Una de dichas voluntades es la voluntad de formación integral de la persona humana (Componentes básicos del Proyecto Académico Pedagógico Solidario, 2011: 145).

Existe la universidad para ofrecer servicios educativos que potencien las competencias y posibilidades de los seres humanos. Es propósito institucional que la comunidad académica toda, desarrolle un aprendizaje significativo, que le permita potencializarse como persona para apoyar y potenciar el desarrollo de las comunidades en que la comunidad académica se inserta. El proceso de educación integral exige la creación de espacios de crecimiento personal y de interacción formativa, creativa y productiva de forma tal que la comunidad académica al tomar conciencia de si misma y de sus múltiples posibilidades, se interese por desarrollar sus propios intereses y así provea formas de vida para si misma y para su pertinencia social, afianzando su identidad personal, cultural y comunitaria (UNAD, 2008).

\section{El emprendimiento desde el currículo}

Para las comunidades académicas es bien sabido que el currículo determina la acción pedagógica y es el resultado de la integración dinámica de los diferentes componentes educativos, pedagógicos y organizacionales de una institución educativa. Busca por lo tanto el currículo, llevar a la comunidad académica a apropiarse críticamente de la realidad, a construir conocimiento de manera propositiva y a que dicho conocimiento pueda ser aplicado en la praxis para la propia transformación de la persona y la transformación de la comunidad, a partir de transferencias propositivas y significativas, del conocimiento desarrollado.

La UNAD en su proceso de transformación continua, se encuentra en este momento construyendo ya no currículos planos sino mapas curriculares que le permitan a la comunidad académica «navegar» por el recorrido de su propia formación integral. UNAD 2011. De ahí pues, que la UNAD en los mapas curriculares que presenta a la comunidad académica, abre las posibilidades para que los procesos académicos se afiancen con otras formas de educación y de participación formativa. Es así como desde la visión de lo internacional, del desarrollo regional y comunitario y desde los servicios de Bienestar Institucional, se planean momentos de formación y de integraciones académicas y pedagógicas, que complementan el currículo formalmente académico, con currículos que buscan afianzar la integralidad de la gestión educativa de la institución.

\section{La UNAD y el trabajo con las comunidades en riesgo de vulnerabilidad}

La Misión de la UNAD sustenta el ofrecimiento de un servicio de educación para todos. En este orden de idea, no podemos perder de vista que al ser un Proyecto Público Vital, la UNAD favorece alianzas para llegar con sus servicios educativos integrales y de calidad a las comunidades que se encuentran en mayor riesgo de vulnerabilidad. De ahí que el $95 \%$ de la comunidad estudiantil de la universidad son ciudadanos que se encuentran clasificados en el estrato socio económico 
1 y 2. (Registro y Control Académico, UNAD, 2012). Dicha situación obliga aún mas a la institución, a proveer mecanismos que les permitan a sus estudiantes encontrar soluciones prácticas a sus necesidades de supervivencia y de desarrollo personal, familiar, profesional y comunitario.

De ahí que promover el espíritu emprendedor, el desarrollo de competencias y capacidades para desarrollar ideas de negocios o ideas de proyectos sociales y comunitarios, entre la comunidad estudiantil, es una obligación que se fortalece al entender que muchos de sus estudiantes, si no desarrollan esas habilidades específicas del emprendimiento, encontrarán mayores dificultades tanto para poder continuar con sus estudios, como para poder organizarse cabalmente en su diario vivir. Cada idea de negocio que se gesta a partir del apoyo de la universidad, cada proyecto solidario que permite mejorar las condiciones de vida de comunidades específicas, es una muestra mas de la pertinencia del servicio educativo que se ofrece en la UNAD.

El programa UNAD-BID-FOMIN: fortalecimiento a las Pymes exportadoras.

Para proyectar a la ciudadanía el valor del emprendimiento y de apoyar a la comunidad en sus iniciativas, se firmó un Convenio entre EI BID y la Universidad Nacional Abierta y a Distancia -UNAD-, para que esta institución ejecutara el Programa Integral de Apoyo a las Pymes para el Acceso al Mercado de Estados Unidos, cuyo objetivo general es apoyar a los empresarios de las pymes colombianas para que ajusten sus productos a los requisitos de acceso que exige el mercado de los Estados Unidos.

A su vez, la universidad se encuentra en proceso de adecuar e implementar un pensum de Comercio Exterior en pro del desarrollo y sostenibilidad del Programa, así como la consolidación de una base de datos con los registros de las pymes de todo el territorio nacional (UNAD - PYMEXCOL, 2008).

\section{ECACEN: Sistema Integral de Emprendimiento Social - SIES}

Como se ha visto es imposible pensar en la UNAD como organización, si no se piensa en ella como una organización preocupada por facilitar, promover y articular el «emprendimiento personal y el emprendimiento hacia lo social» de su comunidad académica. De esa idea vital del emprendimiento como valor fundante de la UNAD, de la realidad de trabajar con población en riesgo de vulnerabilidad y de la idea de manejar un currículo integrador, surgen proposiciones desde varios sectores de la organización, que trabajan por la formación para el emprendimiento. Se interesan las Directivas Nacionales, se interesan los Centros de Educación locales y regionales, se interesan los académicos, se interesa el sistema de consejería, se interesan estudiantes, se interesan los docentes y se interesan hasta los familiares de quienes conforman la comunidad Unadista.

Es así como desde la Escuela de Ciencias de la Administración, Contables, Económicas y de Negocios - ECACEN-, se inicia un proceso de recopilación de las diferentes propuestas que van surgiendo en los diferentes Centros de la UNAD a lo largo y ancho del país. Se encuentra por ejemplo en la Zona Sur, desde el Centro de Palmira como se venía trabajando con el Fondo Emprender del SENA, apoyando estudiantes a presentar sus proyectos o ideas de negocio para que fueran soportadas por dicho Fondo. También en la Zona Boyacá, se estaba gestando un Centro de Pensamiento sobre el Emprendimiento, en la Zona Caribe, en el CEAD Simón Bolívar, desde el 2006, en el momento de su creación, se había constituido el Centro de Desarrollo Empresarial - CEDEM y se había propuesto desde el 2008, la constitución y desarrollo de la Cátedra para el Emprendimiento Social «Alberto Araujo Merlano». Además, en Valledupar como apoyo al CEDEM y otros Centros donde se impulsa el emprendimiento, se está generando un espacio para la constitución 
de una Unidad de Emprendimiento Virtual, al que puedan ingresar la comunidad académica nacional y encontrar el apoyo necesario para generar una idea de negocio, o el montaje y desarrollo de un proyecto específico.

Es así como sirviendo de catalizador del «emprendimiento» como espíritu fundante de la universidad y de las diferentes manifestaciones que en la universidad se presentan sobre el tema de cómo fortalecer el valor del emprendimiento en la comunidad académica, en el 2010 desde la Escuela de Ciencias Administrativas, Contables, Económicas y de Negocios- ECACEN-, surge la creación del Sistema Integral de Emprendimiento Social - SIES. Aprobado por el Consejo Académico el 4 de mayo de 2010 mediante acta de sesión ordinaria No. 004.

El Objetivo General del SIES: «Mejorar el proyecto de vida de nuestros estudiantes en beneficio del desarrollo de la sociedad, a través de la asesoría y gestión de recursos y oportunidades empresariales, laborales y académicas» (Plan de Trabajo del SIES, 2013), pretende proyectar, fortalecer y propiciar frutos para los intereses de emprendimiento de la comunidad académica. Es así como se constituyen fractales de emprendimiento en las Zonas y en los centros locales de la universidad y se acogen y potencias las iniciativas que desde varios centros se estaban favoreciendo en ese sentido. Se fortalecen las Ferias Empresariales y se desarrollan Diálogos de Saberes que buscan brindar oportunidades de intercambio de la comunidad académica con destacadas personalidades del emprendimiento social y comunitario.

\section{Dos casos específicos de puesta en práctica} del valor del emprendimiento en la UNAD

Como se registra anteriormente, en la UNAD son múltiples los casos y las experiencias de puesta en práctica del valor del emprendimiento. En este artículo se muestran resultados de dos casos específicos con la intención de docu- mentar como los esfuerzos que se dan al respecto, brindan beneficios a la comunidad académica Unadista.

\section{Centro de Desarrollo Empresarial-CEDEM}

El papel que juegan las universidades es básico en el impulso y desarrollo del emprendimiento, fortaleciendo la creatividad y la productividad de su comunidad académica. El mundo académico abre oportunidades de innovación a partir de la investigación y generación de conocimiento, aporte básico para la sociedad, porque estimula la creación de empleo y riqueza. «El Emprendimiento como Eje Formador de los estudiantes de la UNAD en Cartagena» (Babilonia, Cure, Pretelt, Álvarez, 2010).

Estas prácticas tan necesarias en la academia, se comienzan a fortalecer en el CEAD Simón Bolívar de la UNAD en Cartagena, a través de la creación de un Centro de Desarrollo Empresarial, que se convierte en un espacio para el desarrollo del emprendimiento, vinculado al SIES de la universidad.

EI CEDEM, asesora planes de negocios y proyectos productivos, participa en convocatorias para favorecer a los mismos emprendedores adscritos al centro, realiza ferias empresariales, convenios con corporaciones de microcrédito, se fundamenta en la Cátedra de Emprendimiento Social y realiza Diálogos de Saberes, estrategias y gestiones todas, que buscan apoyar de manera integral la formación de los estudiantes; a la vez se realizan investigaciones y se les ayuda en la consecución de los recursos para financiar sus proyectos (Proyecto del CEDEM, 2006).

Es importante anotar entre los logros promovidos por la gestión del CEDEM: 14 Ideas empresariales apoyadas con crédito por la Fundación Jemcol, 3 por la Fundación Santo Domingo y 3 seleccionados con capital semilla por Colombia Emprende, 1 por el Fondo Emprender y 1 con el visto bueno de la Universidad. 
Uno de los componentes del Centro de Desarrollo Empresarial, desde lo académico, es la investigación, es así, como se da la participación de estudiantes en «Redcolsi» en sus encuentros locales y nacionales con microempresas en marcha. Se ha conseguido un Reconocimiento Nacional dentro de la Categoría «Mejor empresa en marcha» a la Estudiante de Tecnología en Gestión comercial y de Negocios, Sulays Pérez, con su microempresa «La casita de Papel».

\section{Cátedra de Emprendimiento Social «Alberto Araujo Merlano»}

\section{«Es importante que conozcamos \\ el talento predominante de las \\ personas, esto le permite al ser desarrollarse a plenitud»}

(Merlano, 2010)

La UNAD como una institución de Educación Superior, centrada en la formación en valores, comprometida con el Desarrollo Regional y Comunitario, contribuye a la recuperación del Tejido Social, a la generación de espacios laborales, a la participación ciudadana y a su desarrollo autónomo, lo anterior permite construir y fomentar la Cátedra de Emprendimiento Social «Alberto Araujo Merlano», en honor a un empresario, quien se ha distinguido por los grandes aportes que le ha brindado al país, especialmente a la Región Bolivarense y a su ciudad natal, Cartagena de Indias, desde los diferentes cargos desempeñados en organizaciones gubernamentales, empresariales, del sector productivo y de las entidades sin ánimo de lucro, en las que imprimiéndoles el sello del espíritu emprendedor que lo caracteriza, ha logrado grandes avances, los cuales le han llevado a destacarse a nivel Nacional, Regional y Local.

La cátedra de Emprendimiento, aborda temas como: Emprendimiento, Cultura Empresarial, Espíritu Emprendedor, Productividad, Eco- nomía Solidaria, Desarrollo Regional, Redes Empresariales, entre otros temas; desarrollando estrategias metodológicas presenciales y virtuales: Conferencias, Talleres, Foros, Lecturas Críticas.

\section{Propósitos de la cátedra de emprendimiento social:}

- Poner al alcance de estudiantes, personal académico, instituciones, empresas, una fuente de consulta, material bibliográfico con un sentido estrictamente formativo.

- Divulgar investigaciones, experiencias significativas en diversos espacios (foros, conferencias, cursos, talleres) propiciando el intercambio y la confrontación de saberes.

- Ser una herramienta útil para apoyar ideas de negocios, proyectos productivos, sociales y comunitarios con estrategias de coordinación, capacitación y asesorías, a través de unidades de emprendimiento adscritas al SIES.

\section{La Cátedra tiene como propuesta tres componentes:}

Formar Estudiantes con alto sentido creativo y de ética empresarial que le permita desenvolverse con eficacia y eficiencia en un campo laboral productivo.

Creación de espacios de reflexión, debates y construcción para profundizar los Ejes temáticos, que inciten al conocimiento y aplicación de la Cultura Empresarial, realizando intercambios de experiencias con personas idóneas en los temas propuestos. Además, permitirá la participación activa de Entidades Educativas y Empresariales que inviten y contribuyan al desarrollo de su entorno, en los aspectos: Económicos, Administrativo, Político, Cultural y Tecnológico.

Promover intercambios a nivel internacional que permitan a la Comunidad Unadista, conocer diferentes visiones del mundo de los negocios y del emprendimiento social. 


\section{Conclusión}

Para concluir es importante recalcar que aunque las instituciones de educación superior contemplan el fomento del emprendimiento social, desde una visión-acción de proyección y extensión social, y aportan al tema desde seminarios, talleres, cursos de corta duración, entre otros, son pocas las instituciones que incluyen el emprendimiento social dentro de sus mallas curriculares o desarrollan proyectos de investigación orientados en este tema. Pareciera que la falta de un concepto o idea general de la razón de ser para la formación desde la academia para el emprender social, mueven a las instituciones a propiciar el tema del emprendimiento o como un proceso para generar empresa o como un proceso de desarrollo comunitario pero no como un proceso vital para la información integral (Ortiz, Marulanda, Cañas \& Mesa, 2012).

Por lo tanto se espera que la lectura de este artículo sirva para profundizar en la razón de ser del emprendimiento como un valor sustancial para el desarrollo de la humanidad, por lo tanto, en las instituciones educativas, debe ser tenido siempre en cuenta, como sucede en la UNAD, para la formación integral de las comunidades educativas. El valor del emprendimiento nos lleva a la acción, a la mejora y a la producción. La propuesta es también, que el emprendimiento se reconozca como un valor esencial en el desarrollo de la comunidad Unadista.

Colombia es un país con un enorme potencial y desde las universidades colombianas se ha de trabajar por fomentar, propiciar y formar a los colombianos con nuevas y mejores competencias para asumir los retos que dicho potencial nos ofrece, en especial en las universidades públicas, que como la UNAD, tienen clara la misión de ofrecer educación para todos y en especial de manera incluyente, a la población inmersa en riesgos de gran vulnerabilidad.
La UNAD a partir del valor del emprendimiento, que como se mostró, se encuentra enraizado en toda su estructura organizacional y académica, debe propender y emprender acciones innovadoras, para ampliar cada vez mas, la cobertura de sus servicios educativos de alta calidad, llegando intencionalmente a partir de múltiples mecanismos, a las poblaciones más vulnerables y apartadas del país, y así trabajar apoyándolas para que a partir de la formación integral que se les ofrece, se forme una población activa, dinámica, emprendedora, innovadora, lista para la transformación personal, la transformación profesional y la transformación comunitaria y de la sociedad colombiana.

\section{Referencias}

Babilonia, A., Cure, V., Pretelt, C. \& Álvarez, T. (2010). El Emprendimiento Como Eje Formador De Los Estudiantes De La UNAD en Cartagena. Cartagena: CEAD Simón Bolívar.

Brunner, J. (1984). Acción Pensamiento y Lenguaje. España: Alianza Editorial.

Huetteman, L. (2012). The Value of core values: Five keys for the success through values centered leadership. Valrico, FL 33596: Fidelis SDG, LLC. Recuperado de www. amazon.com

Ley 1014 (2006). De fomento a la cultura del emprendimiento. Bogotá: Diario Oficial No. 46.164 de 27 de enero de 2006.

Departamento Nacional de Planeación (2006). Igualdad de Oportunidades para la prosperidad Social. Bogotá: PND (2006- 2010). Recuperado de: https://www. dnp.gov.co/LinkClick.aspx?fileticket=J7HMrzUQfxY\%3 d\&tabid $=1238$

Ministerio de Educación Nacional (2011). Cartilla para el Emprendimiento en las Instituciones Educativas. Bogotá: MEN. Recuperado de http://www.mineducacion.gov. co/1621/articles-307150 archivo pdf guia39.pdf

Piaget, J. (1947). La Psicología de la Inteligencia. España: Editorial Crítica. 
RedSUMA (2012). El Emprendimiento social en Colombia. Recuperado de: http://www.redsuma.org/emprendimiento-social-en-colombia/el-emprendimiento-socialvisto-desde-la-experiencia-las-universidades/

RedSUMA (2013). El Emprendimiento Social Desde Las Universidades. Recuperado de http://www.redsuma.org/ emprendimiento-social-en-colombia/el-emprendimientosocial-visto-desde-la-experiencia-las-universidades/

Universidad Nacional Abierta y a Distancia (2006). Proyecto Centro de Desarrollo Empresarial -CEDEM. Cartagena: CEAD Simón Bolívar.

Universidad Nacional Abierta y A Distancia (2010). Convenio BID- FOMIN. Bogotá: UNAD. Recuperado de http://www.unadpymexcol.org.

Universidad Nacional Abierta y A Distancia (2012). Estatuto Organizacional según Acuerdo 0030. Bogotá: UNAD. Recuperado de http://www.unad.edu.co/secretaria/remos downloads/sec ACUERDO \%2037 de 27 de\%20Agosto de 2012\%20(2).pdf.

Universidad Nacional Abierta y A Distancia-UNAD (2006). Modelo Pedagógico Integrado- Bases para la Construcción de una Cultura Pedagógica Solidaria. Bogotá: UNAD

Universidad Nacional Abierta y A Distancia (2013). Plan de Trabajo del Sistema Integral de Emprendimiento social-SIES. Bogotá: UNAD.

Universidad Nacional Abierta y a Distancia-UNAD (2011). Proyecto Académico Pedagógico Solidario 3.0. Bogotá: UNAD. Recuperado de http://thumano.unad.edu.co/portal/documentos/induccion/PAP\%20solidario\%20v3.pdf

Universidad Nacional Abierta y A Distancia (2013). Plan de Trabajo del Sistema Integral de Emprendimiento social-SIES. Bogotá: UNAD. 